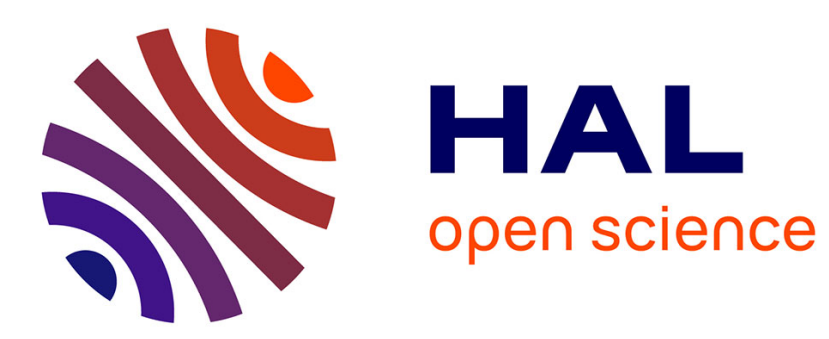

\title{
On thermokinematic analysis of pipe shaping in cast ingots: A numerical simulation via FDM
}

Ugo Andreaus, Francesco Dell'Isola

\section{To cite this version:}

Ugo Andreaus, Francesco Dell'Isola. On thermokinematic analysis of pipe shaping in cast ingots: A numerical simulation via FDM. International Journal of Engineering Science, 1996, pp.19. hal00497337

\section{HAL Id: hal-00497337 \\ https://hal.science/hal-00497337}

Submitted on 4 Jul 2010

HAL is a multi-disciplinary open access archive for the deposit and dissemination of scientific research documents, whether they are published or not. The documents may come from teaching and research institutions in France or abroad, or from public or private research centers.
L'archive ouverte pluridisciplinaire HAL, est destinée au dépôt et à la diffusion de documents scientifiques de niveau recherche, publiés ou non, émanant des établissements d'enseignement et de recherche français ou étrangers, des laboratoires publics ou privés. 


\title{
ON THERMOKINEMATIC ANALYSIS OF PIPE SHAPING IN CAST INGOTS: A NUMERICAL SIMULATION VIA FDM
}

\author{
UGO ANDREAUS and FRANCESCO DELL'ISOLA \\ Dipartimento di Ingegneria Strutturale \& Geotecnica, Facoltà di Ingegneria, Università degli Studi di \\ Roma 'La Sapienza', Via Eudossiana, 18-00184 Roma, Italy
}

(Communicated by G. A. MAUGIN)

\begin{abstract}
Abetract-A model is proposed to describe the influence of solidification shrinkage on the final shape of cast ingots. We regard it as a starting point towards the modelling of the phenomena producing thermal stresses. The model is applied to 'nearly' purely thermal solidification processes occurring in 'long' cylindrical molds. In this instance evolution equations are greatly simplified: their solution in a closed form is found in some technologically interesting instances by means of a semi-inverse method. This solution is used to validate the numerical procedure conceived for tracking the free moving boundary and for determining the final shape of the ingot in a wider range of temperature and heat flux boundary conditions. The above mentioned procedure is based on a nonconventional space-time finite difference discretization of the governing field and boundary equations. A locally uniaxial discretization in space is worked out along either direction of an orthogonal two-dimensional grid. It is refined by iteratively solving the sequence of two uniaxial linear problems, each of which exhibits a narrowly banded coefficient matrix: thus their solutions are found via a factorization algorithm. Copyright (C) 1996 Elsevier Science Ltd
\end{abstract}

\section{INTRODUCTION}

In [1] the phenomenology of solidification processes is discussed: melting and solidification problems occur in numerous important areas of science, engineering and industry. For example, freezing and thawing of foods, production of ice, ice formation on a pipe surface, solidification of metals and chemical reactions all involve either a melting or solidification process. Mathematically, melting/solidification problems are special cases of free moving boundary problems (FMBPs). One of the most interesting classes of phenomena considered in [1] concerns the effects of solidification modalities upon the final shape and mechanical properties of cast ingots. In [2] a list of unsolved or partially solved problems arising in engineering applications is discussed, the aim of that paper being twofold: (i) to draw the reader's attention to some problems which are really relevant for their importance in applied sciences and technology, and (ii) to show how-if engineering answers to practical problems are required-the actual state-of-art makes necessary a compromise in both mathematical rigor and accuracy in the representation of physical reality.

The aim of the present paper is to use the pragmatic approach defended in [2] to face one technologically important problem (see [1]): finding a model suitable for the description of slow solidification processes of cast ingots.

This model is obtained using the results found in [3] and [4], where the methods of continuum theory $[5,6]$ are extended to describe multi-phase systems with interfaces. However, the complete solution of mathematical problems arising when many thermomechanical phenomena are considered, is far from being attained. This circumstance is related to the 'impossibility' results found in [7]: the scope of classical analysis hardly includes FMBPs in more than 1D. Therefore we start a sequence of more and more particularizing hypotheses restricting the scope of applicability of the proposed model but trying to preserve the main features of the physical phenomenon to be described.

We finally consider 2D 'nearly purely thermal' or 'thermokinetic' processes of solidification. Using a semi-inverse method we obtain a quantitative description of those thermokinetic 
solidification processes occurring under some well-defined externally imposed conditions. This description becomes qualitative in nature when quoted conditions are not strictly controlled. Indeed the features of the particular class of solution which we have determined are most probably common to the solutions pertaining to more general cases. Hence some engineering answers can now be guessed using a somehow more rational argument than those available before. Such an approach (nowadays also implemented by means of numerical methods) was frequently and successfully exploited in engineering sciences: the best known case is represented by the intensive exploitation in the theory of structures of the Saint-Venant semi-inverse method. In particular our analysis allows the determination of (i) the scope of some classical semi-empirical rules (e.g. Chvonorinov rule about solidification time), and (ii) some features of the shape of cast ingot. Indeed the analytical solution we have foundassuming that (i) the mold has rectangular cross-section, and (ii) latent heat is only extracted from its vertical sides-shows that the pipes formed in the completely solidified ingot are exactly as deep as the mold, even in the presence of very small differences in mass density between the phases. This circumstance leads us to deduce that, in general, one should expect the formation of deep pipes if latent heat is extracted mainly from lateral sides. Similar considerations have guided the development of the numerical code we use to determine the final shapes of cast ingots in a wide range of heat extraction modalities. It seems that it could be easily improved, in order to 'handle' more physically accurate evolution equations, and is tested upon determined analytical solution. Such a test is not always possible as there are only very limited exact solutions to FMBPs which are concerned with allowable initial conditions and boundary conditions.

As far as front-tracking methods are concerned, finite-difference methods and the finite element technique have been used extensively for the numerical solution of FMB problems. Crank [8] examines a selection of methods for computational efficiency and accuracy, programming complexity, and ease of generalization to more complicated problems. These methods compute, at each step in time, the position of the moving boundary. If the solution is computed on a fixed grid in the space-time domain, the boundary will in general be between two grid points at any given time. Hence special formulae are needed to cope with the PDE and their boundary conditions, in the neighborhood of the free moving boundary (FMB). These formulae must allow for unequal space intervals. Implicit finite difference formulae are used [9] and, since the position of the FMB is not known at the new time, an iterative procedure is inevitable. Alternatively, the grid itself is somehow deformed, or some transformation of variables adopted, so that the FMB is always on a grid line or is fixed in the transformed domain; special averaging procedures are to be used in the element containing the moving boundary, if the problem is discretized via adaptive meshes. Various ways of modifying the grid were proposed, all aiming to avoid the complication and loss of accuracy associated with unequal space intervals near the moving boundary. Adopting a variable time step approach, rather than using a fixed time step and searching for the boundary, one can decide to determine, as a part of the solution, a variable time step such that the FMB coincides with a grid line in space at each time level. Variable space grid methods keep the number of space intervals between a fixed and a moving boundary constant for all time; thus, the space interval varies in each time step, while the FMB is always on the same grid line. The complications due to the unequal grid size near FMB are avoided by moving the whole uniform grid system with the velocity of FMBs and so transfering the unequal interval to the end of the domain, which is a more tractable region. The space grid is adapted at each time step to construct finite elements in space and time for a non-rectangular grid [10]; a weak form of the heat-flow equation is solved, while elements containing the FMB have special features incorporating discontinuous interpolation [11]. Usually, the position of the FMB is assumed to be linear in a special element, and fixed temperature distributions are assumed on its sides. 
Methods which combine unconditional stability (at least up to 2D) with calculation simplicity are the so-called alternating-direction schemes; these are closely related to methods known as splitting or fractional steps methods $[12,13]$.

In the present work the above mentioned alternating-direction method for multidimensional heat-flow problem and implicit finite-difference approximations on a fixed grid are used to solve two-phase solidification problems in $2 \mathrm{D}$. The developed procedure enables the computation of boundary movement in the directions of both coordinate axes. Standard finite-difference approximations to the heat-flow equation are used at grid points far enough from the solidification boundary. Near the boundary, formulae for unequal intervals are incorporated into the equations. Interpolation formulae of Lagrangian type can be used [14]. To avoid loss of accuracy associated with singularities arising if the FMB is too near a grid point, localized quadratic temperature profiles are used [15]. The derivation of these equations is outlined for the two-dimensional case, numerical experiments are reported for solidification problems when the casting is a long bar of rectangular cross-section with dimensions $L_{1} L_{2}$, for various slenderness ratios $L_{2} / L_{1}$ and fixed boundary conditions. From the thermokinetic point of view the problem described in this paper is a purely two-dimensional task (2D); however the procedure can be quite easily extended to the case of time-dependent boundary conditions, temperature-dependent thermophysical properties, mixed initial-boundary-value problems. The achieved results are in agreement with those obtained by an analytical method (Section 4.3). The advantage of the proposed method is its ability to track the FMB which moves along two coordinate axes even when any coordinate line is not assumed to cut the FMB at most once. We use a simple front-tracking method, i.e. a longitudinal-transversal scheme or implicit method with variable direction, determine the temperature field of the casting and the solidification progress, and calculate the total solidification time [16]. However, in numerical applications considered here, the FMB moves essentially along one coordinate axis, and hence only the associated component of its velocity is assumed nonvanishing.

\section{PHYSICAL AND ENGINEERING ASPECTS OF SOLIDIFICATION PHENOMENA}

In [1] is described how the solidification process influences the properties of solidified ingots. The importance of considered phenomena resides in the need to optimize the ingot production process. We consider solidification of pure metals cast in molds whose surfaces are solidification catalyzers, so that solidification begins exactly close to them. We aim to describe the effects of mass density variations determined by thermodynamic phenomena. They occur (1) in phase transition processes, being more relevant, and (2) in both liquid and solid phases, being not completely negligible.

In case (1) the volume variation between solid and liquid phases leads to the formation of empty regions (cavities or pipes) in the mold.

In case (2) in liquid phases convective motions arise, while in solid ones a thermal strain is induced, leading either to plasticity phenomena or to residual stresses or both (cf. p. 230 of [1]). In fact, if the shape of the mold does not allow uniform shrinkage of solidifying or solidified metal the resulting configuration of the ingot is not stress free.

While their volume is determined by the difference of liquid and solid mass density, the shape of cavities and pipes depend at least on (in decreasing order of importance, cf. [1]): (i) relative rate of heat extracted from the bottom and the sides of the mold, (ii) the thermoconvective motions arising in the liquid phase during the solidification, (iii) the field of residual stress arising in the solid phase (see [17]), and (iv) the interfacial phenomena occurring in the considered physical system. 
The a priori determination of the effects of these phenomena is important in engineering applications: to control the quality of the final product one must avoid formation of cavities, determine the placement of pipes, minimize residual and thermal stresses, and limit the volume of regions where plasticity occurs.

We do not try to model all listed phenomena: we use the results found in [3] and [4] in view of the applications which, for the time being, seem possible. We model all the interfaces present in the system simply by means of discontinuity surfaces to which no physical properties are attached. In particular we will not be able to forecast the effects of surface tension on the mechanical properties (cf. pp. $291 \mathrm{ff}$. of [1]) of the solidified ingot. Instead (see Section 4), we can describe 'nearly purely thermal' or 'thermokinematic' 2D solidification.

More particularly, we consider molds whose shape is very 'long' (i.e. a cylinder whose axis is much longer than the diameter of the section), so that the solidification phenomena is described by specifing what occurs in a $2 \mathrm{D}$ section of the molds. The importance of this case in the applications is proved by the interest paid in [18] to $2 \mathrm{D}$ problems in the modelization of continuous casting.

\section{FORMULATION OF THE MODEL}

In the first column of the following table we name the compact surfaces which model the placement of the interfaces specified in the second column:

$\begin{array}{ll}\Lambda \mathrm{A} & \text { liquid phase-surrounding ambient } \\ \Sigma \mathrm{A} & \text { solid phase-surrounding ambient } \\ \Lambda \mathrm{K} & \text { liquid phase-catalyzing inner surface of the mold } \\ \Sigma \mathrm{K} & \text { solid phase-catalyzing inner surface of the mold }\end{array}$

All quoted compact surfaces can move during solidification. Therefore enormous mathematical difficulties arise when a complete set of evolution equations (EE) for all the fields describing the state of considered systems is formulated. Indeed a FMBP for EE is to be formulated where five free moving boundaries appear. Let $K$ be the whole catalyzing inner surface of the mold. We have

$$
\Lambda K \cup \Sigma K \subseteq K
$$

We assume that:

(H1) $K$ is a fixed known surface;

(H2) there exists a fixed compact domain $D$ (in the sense of Truesdell [5]) in which the solidification process occurs whose boundary $\partial D$ includes (and eventually coincides with) $K$.

In processes occurring in the absence of gravity $K$ is closed, in order to bound the domain $D$, while in the presence of gravity the 'upper' part of $\partial D$ includes the interface solidifying metal-surrounding ambient (e.g. air or supplementary refrigerating system).

In the absence of an external supply of liquid metal, $\Sigma A$ is not empty and does not move anymore due to solidification. Indeed, because of mass density change occurring in phase transition, (i) the liquid phase in general is not covering all the boundary of $S$ not in contact with $K$, and (ii) a flow arises in the liquid phase at least to assure mass balance.

We call $S$ and $L$ the domains occupied by liquid and solid phases. These sets are time 
dependent. We assume that the state of the considered system is determined if the following fields are known:

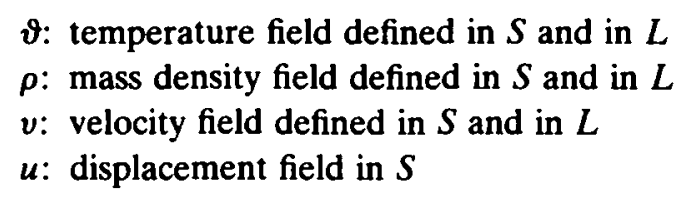

We remark that a problem arises when defining the displacement field in the solid phase, as no global reference configuration can be introduced for a solid which before the process did not exist. Following what is usually assumed in the literature (for instance see [17]) we regard $u$ as the displacement from the place occupied by the substantial solid particle at the instant in which it became solid. We can formulate for the fields (3.2) the EE local consequences of the corresponding global balance laws. We denote with an upper dot the material time derivative, with $n$ the unit normal vector field to considered surfaces whose normal speed is $c_{n}$ and with the symbol $[F]_{\varphi}, \varphi=s, 1$ the limit value of the field evaluated on the considered surface respectively from the solid and liquid side.

Balance of mass

$$
\begin{gathered}
\dot{\rho}+\rho \operatorname{div} v=0 \text { in } S \cup L ; \int_{S} \rho+\int_{L} \rho=M \\
\left\{\left[\rho\left(v \cdot n-c_{n}\right)\right]_{\mathrm{s}}-\left[\rho\left(v \cdot n-c_{n}\right)\right]_{\}}\right\}=0 \text { on } \Sigma \Lambda
\end{gathered}
$$

Equations (3.3) and (3.4) ensure that there is no mass production inside bulk phases and at the $\Sigma \Lambda$ interface; the total mass $M$ of the solidifying specimen will be assumed constant.

Balance of force

$$
\rho \dot{v}=\operatorname{div} T+b \text { in } S \cup L
$$

where $T$ is the Cauchy stress tensor and $b$ is the volume density of external force;

$$
\left\{\left[\rho v\left(v \cdot n-c_{n}\right)-T n\right]_{s}-\left[\rho v\left(v \cdot n-c_{n}\right)-T n\right]_{1}\right\}=0 \text { on } \Sigma \Lambda .
$$

Equation (3.6) holds because of the absence of surface tension and related phenomena. We then add the following boundary condition:

$$
c_{\mathrm{n}}=0 \text { on } \Sigma A \cup \Sigma K ; T n \text { assigned on } \Lambda A
$$

stating that (i) solid-ambient, solid-catalyzer, liquid-catalyzer interfaces do not move, and (ii) external ambient exerts an assigned contact force on the liquid phase.

\section{Balance of energy and entropy}

If $\epsilon$ is the intemal energy per unit mass and $h$ is heat flux we have that:

$$
\begin{gathered}
\rho \dot{\epsilon}=T: \operatorname{grad} v+\operatorname{div} h+r \text { in } S \cup L \\
\left\{\left[\rho\left(\frac{v^{2}}{2}+\epsilon\right)\left(v \cdot n-c_{n}\right)-v T n-h \cdot n\right]_{\mathrm{s}}-\left[\rho\left(\frac{v^{2}}{2}+\epsilon\right)\left(v \cdot n-c_{n}\right)-v T n-h \cdot n\right]_{1}\right\}=0 \text { on } \Sigma \Lambda .
\end{gathered}
$$

Moreover we assume that $\vartheta$ or $h$ are assigned at every point on $\Sigma A \cup \Sigma K \cup \Lambda K$. These 
conditions determine how the solidification process is controlled. Finally we assume that on $\Sigma \Lambda$ no entropy dissipation occurs:

$$
\left\{\left[\rho \eta\left(v \cdot n-c_{n}\right)-\frac{h \cdot n}{\vartheta}\right]_{\mathrm{s}}-\left[\rho \eta\left(v \cdot n-c_{\mathrm{n}}\right)-\frac{h \cdot n}{\vartheta}\right]_{1}\right\}=0 \text { on } \Sigma \Lambda .
$$

Some initial conditions have to be added to the listed equations, together with all constitutive equations, in order to well-pose the mathematical problem representing the searched model.

To our knowledge this problem is not solved: no general theorem of existence and uniqueness seems to be available and no known numerical method (see [19]) seems to be comprehensive enough to be applicable in the considered instance.

\section{THERMOKINEMATIC 2D-SOLIDIFICATION: EXACT SOLUTIONS BY MEANS OF A SEMI-INVERSE METHOD}

We call a process thermokinematic or nearly purely thermal 2D-solidification if the following hypotheses are verified:

(H1) Solid and liquid phases are incompressible and their densities are uniform and constant. We call $\rho_{\mathrm{L}}$ and $\rho_{\mathrm{S}}$ respectively the liquid and solid phase mass densities.

(H2.1) Some symmetry considerations reduce the problem to a two-dimensional one: the considered mold has the shape of a long beam with constant section. Then the spatial coordinates reduce to $\left(x_{1}, x_{2}\right)$ and the surfaces modelling the interfaces can be determined by curves in the plane of the section.

(H2.2) The section of the mold is rectangular and its sides are parallel to the $\left(x_{1}, x_{2}\right)$ axes. Let $\gamma$ be the curve representing the union $\Sigma A \cup \Sigma \Lambda$. Latent heat is extracted mainly from the lateral (i.e. parallel to the $x_{2}$-axis) side of the mold in such a way that $\gamma$ can be determined always during the solidification process as the graph of a function $f$ of the variable $x_{2}$.

(H3) When phase transition occurs the total volume of the present matter varies because of the mass density difference between the phases. In solidification processes the volume decreases and because of the mass conservation law a velocity field has to arise in the liquid phase in order to allow such a volume change. This velocity field has negligible effects on all thermomechanical phenomena: it only assures the global balance of mass. Moreover the solidification process is slow enough to allow the liquid phase to flow in an equilibrium configuration once a new solid part is formed, and before the solidification process is able to subtract to the liquid phase again a relevant quantity of mass. This means that $\Lambda A$ interface is flat and parallel to $x_{1}$-axis (i.e. horizontal): its level $h$ has to be determined by the global balance of mass. We assume that convective velocity fields are negligible.

(H4) The external ambient exerts a pressure $p_{\mathrm{e}}$ on the solidifying system and

$$
p_{\mathrm{e}} \gg\left(\rho_{\mathrm{S}}+\rho_{\mathrm{L}}\right)\left(l_{1}+l_{2}\right) g
$$

so that stress tensor inside the liquid and solid phase is equal to $p_{\mathrm{e}} I$.

4.1 Relation between the level $h$ of liquid phase and the solidified phase profile $f$. The case of flat $\Sigma \Lambda$

If $(\mathrm{H} 2)$ and $(\mathrm{H} 3)$ are verified the global balance of mass implies:

$$
F(h ; f(\cdot)):=\int_{0}^{h} \Delta \rho f(y) \mathrm{d} y+\int_{h}^{l_{2}} \rho_{\mathrm{S}} f(y) \mathrm{d} y+h l_{1} \rho_{\mathrm{L}}-M=0
$$

where $\Delta \rho:=\rho_{\mathrm{S}}-\rho_{\mathrm{L}}, l_{1}$ and $l_{2}$ are the lengths of the sides of the mold parallel to the $x_{1}-$ and 
$x_{2}$-axes and $F$ is a function of $h \in\left[0, l_{2}\right]$ and a functional of the profile $f$ of the solidified profile. As

$$
F(0 ; f)<0 ; F\left(l_{2} ; f\right)>0 ; \partial F / \partial h>0
$$

then for every profile $f$ there exists a unique level $h$ verifying (4.1). For a solidification process, a family of solidified profiles has to be considered. We then introduce a family of functions $f$ depending on the time variable $t$ describing the profile at instant $t$. A particular solution for EE is obtained assuming that:

(H5) the part of $\gamma$ representing the interface $\Sigma \Lambda$ is, at every instant $t$, a vertical straight line.

If hypothesis (H5) is verified and if $T$ is the solidification time, then $f\left(x_{2}, T\right)$ is easily obtained using equation (4.1) and the condition $f\left(l_{2}, T\right)=0$. Indeed we have:

$$
f\left(x_{2}, T\right)=l_{1}\left(1-\left(\frac{x_{2}}{l_{2}}\right)^{\alpha}\right)
$$

with $\alpha=\rho_{2} / \Delta \rho$.

The proof of (4.3) is easily obtained as follows. Consider for every $a \in\left[0, l_{2}\right]$ the function $g_{a}\left(x_{2}\right)$ defined in the interval $\left[0, l_{2}\right]$ as follows:

$$
g_{a}\left(x_{2}\right):=\left\{\begin{array}{l}
f\left(x_{2}, T\right) \text { if } x_{2}>a \\
f(a, T) \text { if } x_{2}<a .
\end{array}\right.
$$

REMARK. When (H5) is verified, the function $g_{a}$ represents the solidified profile when the level of liquid phase is equal to $a$. Equation (4.4), means that once one part of the solidified profile is no more in contact with the liquid phase it will not evolve.

Then, using $M=\rho_{\mathrm{L}} l_{1} l_{2}$ and (4.1) we have:

$$
\rho_{\mathrm{s}}\left\{\int_{a}^{l_{2}} f\left(x_{2}, T\right) \mathrm{d} x_{2}+a f(a, T)\right\}=\rho_{\mathrm{L}}\left\{a f(a, T)+l_{1}\left(l_{2}-a\right)\right\} .
$$

REMARK. Equation (4.5) can be interpreted as follows: the mass of solidified phase is equal to the mass of the 'disappeared' liquid.

Deriving equation (4.5) with respect to the variable $a$ we obtain the ODE whose solution, verifying the condition $f\left(l_{2}, T\right)=0$, is given by (4.3).

\subsection{Modalities of extraction of latent heat}

The balance of energy and hypotheses (H1), (H3) and (H4) imply that latent heat is extracted only because of heat conduction and that the derived FMBP is the classical Stefan problem. We report here the Neumann solution for one-dimensional solidification (cf. [8]). Indeed in Section 5 we find numerical solutions for a wide class of $2 \mathrm{D}$ thermokinetic solidification processes to be tested on analytical solutions. Such a solution is found in Section 4.3 by means of a semi-inverse method in which the Neumann solution is needed.

Let $x$ and $t$ be the space and time variables. We consider at $t=0$ the liquid phase occupying the $[0, \infty]$ space interval with uniform temperature $\vartheta_{F}$ (melting temperature). Moreover we assume that for every $t>0$ the temperature at $x=0$ is $\vartheta_{R}<\vartheta_{F}$. Then a solidification process takes place and

(i) the solid-liquid interface moves following the law

$$
\xi(t)=\alpha \sqrt{t}
$$

where $\alpha$ is the solution of the following transcendent equation:

$$
\frac{L}{2} \alpha \sqrt{\pi}=\frac{\left(\vartheta_{R}-\vartheta_{F}\right) K_{1} \exp \left(-\alpha^{2} / 4 a_{1}\right)}{\sqrt{a_{1}} \operatorname{erf}\left(\alpha / 2 \sqrt{a_{1}}\right)}
$$


in which $L$ is volume latent heat, $K_{1}$ is heat conductivity of the solid phase, and $a_{1}:=K_{1} / c_{1}$ with $c_{1}$ denoting volume specific heat;

2. (ii) the temperature field $\vartheta_{1}$ in solid phase is given by

$$
\vartheta_{1}=\vartheta_{R}-\frac{\left(\vartheta_{R}-\vartheta_{F}\right)}{\operatorname{erf}\left(\alpha / 2 \sqrt{a_{1}}\right)} \operatorname{erf}\left(x / 2 \sqrt{a_{1} t}\right)
$$

\subsection{An exact solution by means of a semi-inverse method}

We determine a class of thermokinematic processes assigning some of the necessary boundary conditions and assuming some particular properties of the temperature fields. Analytical expressions for these fields and for the position of the free moving boundaries can be thus determined. We assume that (cf. Fig. 3):

1. at the initial instant $t_{0}$ the interface $\Sigma \Lambda$ is formed on the lateral walls (LWs: i.e. two parallel sides of the rectangular section of the mold) and that the liquid temperature is equal to $\vartheta_{F}$; moreover heat extraction occurs only through LWs, while the remaining contact interface of the liquid with the mold (on the basis) does not allow any heat flux;

2. at every instant $t$, during solidification at the $\Sigma \Lambda$ and $\Lambda A$ interfaces the temperature has the constant value $\vartheta_{F}$; the $\Lambda A$ interface-which is flat and parallel to basis of the section of the mold-does not allow any heat flux;

3. also the $\Sigma \Lambda$ interface is flat and parallel to the LWs of the mold and its distance $\xi$ from the LWs is given by equation (4.6); and

4. at every instant $t$, the solid phase in contact with one of the LWs occupies the region whose intersection with the plane of the section of the mold is bounded by the LWs and the graph of the function defined by equation (4.4) [with $a=\xi(t)$ as given by (4.6)]; moreover the temperature field $\vartheta_{1}$ in the solid phase does not depend on the coordinate $x_{2}$ along the direction of the LWs but only on the coordinate $x_{1}$ along the direction parallel to the mold basis. Thus we obtain equation (4.8) when replacing the variable $x$ with the variable $x_{1}$.

Remarks. (i) As a result of (4.8), the Stefan condition is verified at the $\Sigma \Lambda$ interface.

(ii) the boundary condition at the $\Sigma A$ interface can no longer be assigned, as it is specified by the expression for the temperature field determined in point (4) of the list above. In particular we observe that the temperature field on the interface solid-external ambient is equal to $\vartheta_{R}$ on LWs and to $\vartheta_{F}$ at its intersection with the $\Sigma \Lambda$ interface.

In conclusion we have found a particular solution to the solidification problem formulated in the previous sections. The most striking feature of this solution is implied by the shape of the function $f$ defined by equation (4.3): also a very small mass density difference between the phases-when heat extraction occurs only through the LWs-implies the solidification of two different ingots inside the mold. This can be regarded as a property common to all slow thermokinetic solidification processes: in these processes the shrinkage pipes are very deep.

\section{FINITE DIFFERENCE METHOD FOR THE 2D PROBLEM}

\subsection{Generalities}

Let $\bar{G} \equiv \bigcup_{\varphi} \bar{G}_{\varphi}$ be a two-dimensional domaint in the $\left(x_{1}, x_{2}\right)$-plane, $\Gamma \equiv \bigcup_{\varphi} \Gamma_{\varphi}$ be its outer boundary, and $\Phi$ be the FMB between two homogeneous phases: a solid phase (phase $s$ or

tWe adopt a usual index notation in which Greek minuscules have the values 1,2 and repeated indices do not mean summation. 
$\varphi=1$ ) and a liquid phase (phase 1 or $\varphi=2$ ); these phases are characterized by specific heat $c_{\varphi}$, thermal conductivity $k_{\varphi}$, and mass density $\rho_{\varphi}$ constant in either phase. In either of the above mentioned phases the temperature $\vartheta(x ; t)$ is governed by the thermal conduction equation together with the initial and boundary conditions respectively given in the form

$$
\begin{gathered}
x=\left\{x_{1}, x_{2}\right\} \in G_{\varphi}, 0<t \leq T, \vartheta_{\varphi}=\vartheta_{\varphi}(x ; t) ; \frac{\partial \vartheta_{\varphi}}{\partial t}=a_{\varphi}^{2} \operatorname{lap}\left(u_{\varphi}\right), a_{\varphi}^{2}=\left(\frac{k}{c \rho}\right) \\
\operatorname{lap}\left(\vartheta_{\varphi}\right)=\Delta \vartheta_{\varphi}=\sum_{\alpha} \frac{\partial^{2} \vartheta_{\varphi}}{\partial x_{\alpha}^{2}}=\sum_{\alpha} D_{\alpha} \vartheta_{\varphi} ; \\
\vartheta_{\varphi}(x ; 0)=\vartheta_{\varphi}^{0}(x), x \in \bar{G}_{\varphi}, t=0,\left.\vartheta_{\varphi}\right|_{\Gamma_{\varphi \varphi}}=\bar{\vartheta}_{\varphi}(x ; t), x \in \Gamma_{\varphi \varphi}, 0<t \leq T \\
-[\operatorname{kgrad}(\vartheta)]_{\varphi} \cdot n_{\Gamma_{\varphi \varphi}}=-\left.k_{\varphi} \frac{\partial \vartheta}{\partial n}\right|_{\Gamma_{\varphi \varphi}}=\bar{q}_{\varphi}(x ; t) \cdot n_{\Gamma_{\varphi \varphi}}, x \in \in_{\Gamma_{\varphi \varphi}}, 0<t \leq T
\end{gathered}
$$

In equations $(5.1 \mathrm{~b}), \Gamma_{\varphi u}$ and $\Gamma_{\varphi q}$ denote the outer boundary regions where temperature $\bar{\vartheta}_{\varphi}$ and heat flux $\bar{q}_{\varphi}$ are respectively assigned; $n_{\Gamma}$ denotes the outer normal to the contour line $\Gamma_{\varphi q}$. For simplicity's sake, in the following we will assume $a_{\varphi}^{2}=1$.

On the FMB $\Phi$ between liquid and solid phases the temperature $\vartheta(\xi ; t)=\vartheta^{*}, \xi \in \Phi$, is constant and equal to the melting temperature $\vartheta^{*}$; the heat fluxes are discontinuous and their difference is $c^{*} \dot{\xi}_{n_{\phi},}$, where $c^{*}$ is the latent heat at melting temperature and $\dot{\xi}_{n_{\phi}}$ is the FMB speed in the direction normal $n_{\Phi}$ to the FMB itself; the normal pointing from solid to liquid phase is taken positively oriented. On the FMB $\Phi$ between liquid and solid phases the following boundary conditions do hold:

$$
\begin{gathered}
\xi \in \Phi, \xi_{\alpha}=\xi_{\alpha}(t), \dot{\xi}_{\alpha}=\frac{\partial \xi_{\alpha}}{\partial t}, \vartheta(\xi ; t)=\vartheta^{*} \\
\left\{[\operatorname{kgrad}(\vartheta)]_{\mathrm{s}}-[\operatorname{kgrad}(\vartheta)]_{\}}\right\} \cdot n_{\Phi}=c^{*} \frac{\mathrm{d} \xi}{\mathrm{d} t} \cdot n_{\Phi}=c^{*} \dot{\xi}_{n_{\Phi}} \\
{[\operatorname{grad}(\vartheta)]_{\alpha}=[\nabla(\vartheta)]_{\alpha}=\frac{\partial \vartheta}{\partial x_{\alpha}}, \vartheta(x ; t)<\vartheta^{*}, x \in \bar{G}_{\mathrm{s}} ; \vartheta(x ; t)>\vartheta^{*}, x \in \bar{G}_{1}}
\end{gathered}
$$

\subsection{Discretization in the space-time domain}

Let the space-time mesh $\varpi_{h_{1} h_{2} \tau}$ (closure of $\omega_{h_{1} h_{2} \tau}$ ) be generated in $\bar{G}=G+\Gamma$ and denoted

$$
\varpi_{h_{1} h_{2} \tau}=\varpi_{h_{1}} \times \varpi_{h_{2}} \times \varpi_{\tau}=\left\{\left(x_{\alpha, i_{\alpha}}=i_{\alpha} h_{\alpha}, t_{j}=j \tau\right) ; i_{\alpha}=0,1, N_{\alpha}, j=0,1, \ldots, N_{0}\right\}
$$

This mesh is uniform in each of the variables $\left(x_{1}, x_{2} ; t\right)$. The mesh $\boldsymbol{\sigma}_{h_{1} h_{2} \tau}$ is comprised, obviously, of the meet point of the planes $x_{a}=x_{\alpha, i_{u}}\left(i_{\alpha}=0,1, \ldots, N_{\alpha}\right)$ and of the planes $t=t_{j}\left(j=0,1, \ldots, N_{0}\right)$. The sets of all the inner and boundary nodes of the mesh $\varpi_{h_{1} h_{2} \tau}$ are respectively denoted

$$
\begin{gathered}
\omega_{h_{1} h_{2} \tau}=\omega_{h_{1}} \times \omega_{h_{2}} \times \omega_{\tau}=\left\{\left(x_{1, i_{1}}, x_{2, i_{2}} ; t_{j}\right), 0<i_{\alpha}<N_{\alpha}, 0<j \leq N_{0}\right\} \\
\gamma_{h_{1} h_{2} \tau}=\left\{\left(x_{1, i_{1}}, x_{2, i_{2}} ; t_{j}\right), i=0,0 \leq j \leq N_{0} ; i_{\alpha}=N_{\alpha}, 0 \leq j \leq N_{0} ; j=0,0 \leq i_{\alpha} \leq N_{\alpha}\right\} .
\end{gathered}
$$

The index $\alpha$ is omitted for the sake of brevity; dividing the range $[0, L]$ into $N$ steps by points $x_{1}<x_{2}<\ldots<x_{N-1}<L$ yields the mesh $\varpi_{h}=\left\{x_{i}, i=0,1,2, \ldots, N, x_{0}=0, x_{N}=L\right\}$, having step $h_{i}=x_{i}-x_{i-1}$ which depends upon the index $i$ of the node $x_{i}$. If $h_{i} \neq h_{i+1}$ for at least one index $i$, then the mesh $\varpi_{h}=\varpi_{h}^{*}$ is called non-uniform. If $h_{i}=$ constant $=h=L / N$ for all $i=1,2, \ldots, N$, we have a uniform mesh. In the irregular nodes of $\varpi_{h_{1} h_{2}}$ the difference operator applies to a non-uniform mesh [14]. The following differential operators should be approximated for the 
problem at hand: the temperature time rate $\frac{\partial \vartheta}{\partial t}$, the temperature space Laplacian operator $\Delta \vartheta$, the temperature space gradient $\nabla \vartheta$, the FMB time rate $\frac{\partial \varphi}{\partial t}$, the normal to the FMB $n_{\phi}$.

So far, a numerical procedure has been developed in order to solve the governing equations with both continuous and discontinuous coefficients. The total number of required calculations, i.e. the total number of arithmetical operations needed to solve the problem at the desired degree of accuracy, turns out to be a crucial point in the multi-dimensional mesh methods. The discretization schemes which combine the better features of the explicit algorithm (total amount of operations proportional to the total number of mesh nodes $\left(N_{1}-1\right) *\left(N_{2}-1\right)$ ) with those of the implicit algorithm (unconditional stability, i.e. for any $\tau$ and $h_{1}, h_{2}$ ) are called economical schemes. This basic idea is embodied in the so-called longitudinal-transversal scheme or implicit method with variable direction. This locally unidimensional method is suitable for use in a broad range of circumstances, namely thermal conduction equation with constant, variable and discontinuous coefficients in an arbitary domain $G$ having any dimension $p$.

\section{PROBLEM DISCRETIZATION}

\subsection{Discretization of the thermal conduction equation}

The temperature time rate $\frac{\partial \vartheta}{\partial t}$ and the temperature space Laplacian operator $\Delta \vartheta$ are to be approximated in the difference scheme discretizing the thermal conduction equation.

An approximated solution $v_{j+1}$ for $t=t_{j+1}$ is generated for equation (5.1a) by successively involving (for $\alpha=1,2$ ) the unidimensional equations of thermal conduction with the space-time continuity conditions between discretization directions:

$$
\begin{gathered}
(\alpha=1,2), t^{j} \leq t \leq t^{j+1} \\
\frac{\partial v_{(\alpha)}}{\partial t}=\sum_{\beta=1}^{2} D_{\beta} v_{(\beta)}, \\
v_{(\alpha-1)}^{j+1}=v_{(\alpha)}^{j}(\alpha=2) \\
v_{(1)}^{j}=v^{j},
\end{gathered}
$$

and the boundary and initial conditions (5.1b). The solution of such a problem is $v^{j+1}=v_{(2)}^{j+1}$. Assigning $v^{0}=\vartheta^{0}(x)$ gives $v^{j+1}$.

In either equation (6.1) having index $\alpha$ we substitute the differential operators $\partial_{t}$ and $D$ with the difference operators $\Lambda_{\alpha}$ and $\Lambda_{\alpha \alpha}$ [20]:

$$
\begin{gathered}
\Lambda_{\imath}^{-} v_{(\alpha)}^{j+1}=\left(\frac{k}{\rho c}\right)_{\varphi}\left[\Lambda_{\alpha \alpha} v_{(\alpha)}^{j+1}+\Lambda_{\alpha-1, \alpha-1} v_{(\alpha-1)}^{j}\right] \\
\alpha=1,2(\alpha=1 \Rightarrow \alpha-1=2) \\
\varphi=1,2 .
\end{gathered}
$$

In equation (6.2), $\varphi=1$ and $\varphi=2$ denote solid and liquid phase respectively. The operator $\Lambda_{\alpha \alpha}$ is substituted by the difference operator $L_{\alpha \alpha}$ in the neighborhood of the moving boundary [14].

\subsection{Discretization of the evolutional equation of $F M B$}

The space gradient operator $\nabla \vartheta$, the FMB time rate $\frac{\partial \varphi}{\partial t}$, and the outward normal $n$ to the FMB are to be approximated in the finite difference scheme discretizing the evolutional 


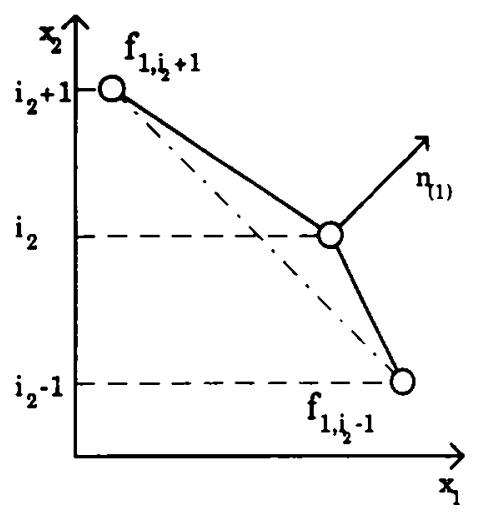

a) Direction $a=1$

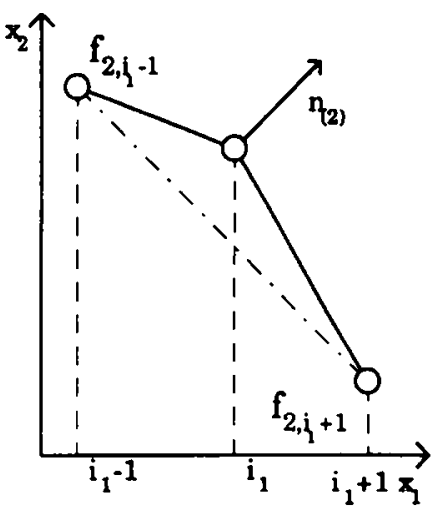

b) Direction $\alpha=2$

Fig. 1. Approximation of the normal to the moving boundary.

equation of the FMB. We denote by $v, s_{(\alpha)}$ and $n_{(\alpha)}$ the approximated solutions of temperature $\boldsymbol{\vartheta}$, FMB $\xi$, and FMB normal $n_{\phi}$, respectively. Solving a unidimensional problem on each row (column) yields the following form of the boundary conditions on the FMB (Fig. 1).

In more detail, the position and velocity vectors of a moving boundary point, and the vector normal to the FMB and pointing from solid to liquid phase have components

$$
\begin{gathered}
\alpha, \delta=1,2,\left\{\begin{array}{l}
\delta \neq \alpha-1 \\
\delta=\alpha-1
\end{array}\right\} \Rightarrow s_{(\alpha) \delta}=\left\{\begin{array}{c}
f_{\alpha}\left(x_{\alpha-1} ; t\right) \\
x_{\alpha-1}
\end{array}\right\}, \dot{s}_{(\alpha) \delta}=\left\{\begin{array}{c}
\dot{f}_{\alpha}\left(x_{\alpha-1} ; t\right) \\
0
\end{array}\right\},(\alpha=1 \Rightarrow \alpha-1=2) ; \\
\alpha, \delta=1,2,\left\{\begin{array}{l}
\delta \neq \alpha-1 \\
\delta=\alpha-1
\end{array}\right\} \Rightarrow n_{(\alpha) \delta}=\left\{\begin{array}{c}
1 \\
-\partial f_{\alpha} / \partial x_{\alpha-1}
\end{array}\right\} ;(\alpha=1 \Rightarrow \alpha-1=2) .
\end{gathered}
$$

The transition temperature and the evolutional equation of the moving boundary are approximated by

$$
\begin{gathered}
\alpha=1,2: v_{(\alpha)}\left(s_{(\alpha)} ; t\right)=\vartheta^{*}, \alpha=1 \Rightarrow \alpha-1=2 \\
{\left[\left(k \frac{\partial v_{(\alpha)}}{\partial x_{\alpha}}\right)_{s}-\left(k \frac{\partial v_{(\alpha)}}{\partial x_{\alpha}}\right)_{1}\right]_{(\alpha) \alpha}+\left[\left(k \frac{\partial v_{(\alpha-1)}}{\partial x_{\alpha-1}}\right)_{s}-\left(k \frac{\partial v_{(\alpha-1)}}{\partial x_{\alpha-1}}\right)_{1}\right] n_{(\alpha) \alpha-1}=c^{*} \frac{\partial f_{\alpha}}{\partial t} n_{(\alpha) \alpha}}
\end{gathered}
$$

The space-time continuity equations between discretization directions read as follows:

$$
v_{(\alpha)}^{j}=v_{(\alpha-1)}^{j+1}, f_{(\alpha)}^{j}=f_{(\alpha-1)}^{j+1}(\alpha=2) ; v_{(1)}^{j}=v^{j}, f_{(1)}^{j}=f^{j} .
$$

Finally, knowledge of $f^{0}=0$ yields $f^{j+1}$. In equations (6.3)-(6.7) and in the following, lower indices within round parentheses denote differentiation direction, whereas Cartesian components are without parentheses.

In each equation (6.5)-(6.7) having index $\alpha$ the differential operator $\partial$ is substituted by the difference operators $L_{\alpha}$ and $\Lambda_{\alpha}[14,20]$ :

$$
v_{(\alpha)}^{j+1}=\vartheta^{*} ; k_{s}\left[L_{\alpha}^{-\ddot{v}} \boldsymbol{v}_{(\alpha)}^{i+1}-\Lambda_{\alpha-1}^{0} v_{\alpha-1}^{j} \Lambda_{\alpha-1}^{0} f_{\alpha}^{j}\right]-k_{1}\left[L_{\alpha}^{+} v_{(\alpha)}^{j+1}-\Lambda_{\alpha-1}^{0} v_{(\alpha-1)}^{j} \Lambda_{\alpha-1}^{0} f_{\alpha}^{j}\right]=c^{*} \Lambda_{t}^{-} f_{\alpha}^{j+1}
$$

The normal to the moving boundary is explicitly approximated by means of the weighted central difference (weighted average of left and right differences, the weight being the layer thickness):

$$
\Lambda^{w}{ }_{\alpha} y_{i}=-\frac{3 h_{i}-h_{i+1}}{2 h_{i}\left(h_{i}+h_{i+1}\right)} y_{i-1}+\frac{h_{i}-h_{i+1}}{2 h_{i} h_{i+1}} y_{i}+\frac{3 h_{i+1}-h_{i}}{2 h_{i+1}\left(h_{i}+h_{i+1}\right)} y_{i+1}
$$

instead of the difference operator $\Lambda^{0}{ }_{\alpha}[20]$ at the 'corner' point where the slope of the moving boundary is abruptly varying near the liquid head. 


\subsection{Discretization of the initial and boundary conditions}

In order to formulate the boundary conditions for $v_{(\alpha)}$, we consider an inner point $x \in \dot{\omega}_{h_{1} h_{2}}$ and we trace through it the straight line $C_{\alpha}$ parallel to the axis $O x_{\alpha}$. We study the simpler case where $C_{\alpha}$ meets $\Gamma$ through two points $P_{\alpha}^{-}$and $P_{\alpha}^{+}$. We denote by $\gamma_{h_{\alpha}}(\alpha=1,2)$ the set of all points $P_{\alpha}^{-}$and $P_{\alpha}^{+}$. If $G=G_{0}=0 \leq x_{\alpha} \leq L_{\alpha}, \alpha=1,2$ is a rectangle, hence $\gamma_{h_{\alpha}}$ is comprised of nodes $\left(i_{1} h_{1}, i_{2} h_{2}\right)$ lying on the sides $x_{\alpha}=0\left(i_{\alpha}=0\right)$ and $x_{\alpha}=L_{\alpha}\left(i_{\alpha}=N_{\alpha}\right), \alpha=1,2$. The alternative boundary conditions for $v_{(\alpha)}$ are obviously assigned only on $\gamma_{h_{\alpha}}$ :

$$
\begin{gathered}
v_{(\alpha)}=\bar{\vartheta}(x ; t) \text { for } x \in \gamma_{h_{\alpha}}, \alpha=1,2 \\
\left\{-\operatorname{kgrad}\left[v_{(\alpha)}\right]\right\}_{\varphi} \cdot\left[\mp e_{(\alpha)}\right]=\left[k \frac{\partial v_{(\alpha)}}{\partial x_{\alpha}}\right]_{\varphi}=\bar{q}(x ; t) \cdot e_{(\alpha)}=\bar{q}_{\alpha}(x ; t) \text { for } x \in \gamma_{h_{\alpha}}, \alpha=1,2
\end{gathered}
$$

where $e_{(\alpha)}$ denotes the unit vector in the $\alpha$-coordinate direction, and the minus/plus sign refers respectively to side $x_{\alpha}=0$ or $x_{\alpha}=L_{\alpha}$.

At the initial time $t=0$ the following condition is given:

$$
v_{(\alpha)}(x ; 0)=\bar{\vartheta}^{0}(x) \text { for } x \in \omega_{h_{1} h_{2}} .
$$

Equations (6.9) and (6.10) uniquely define $v^{\prime}$ for any $i_{\alpha}=1,2, \ldots, N_{\alpha}$ and $x \in \omega_{h_{1} h_{2} \tau}$. More explicitly, equations $(6.9 \mathrm{~b})$ take the form

$$
\pm\left[k \Lambda_{\alpha}^{+}\right]_{\varphi}=\bar{q}_{\alpha}(x ; t) \text { for } x \in \gamma_{h_{u}}, \alpha=1,2
$$

where the differential operator $\partial_{\alpha}$ has been approximated by means of the difference operator $\Lambda_{\alpha}^{+}[20]$.

\section{ITERATIVE METHOD OF SOLUTION}

\subsection{Time discretization}

The jump from time $j$ to time $j+1$ is performed in two steps. First, equations (6.2) and (6.8) are solved for $\alpha=1$ implicitly in the $x_{1}$-direction and explicitly in the $x_{2}$-direction, and then the same equations are solved for $\alpha=2$ explicitly in the $x_{1}$-direction and implicitly in the $x_{2}$-direction. We thus obtain a sequence of locally unidimensional schemes. Typically for the FDM approach this leads to a linear system of equations, which has a general band form; an effective algorithm based on the Gauss elimination which uses certain properties of general band matrices and computer data type architecture can be found in [21, 22]. In the locally unidimensional scheme not all the discretization directions exhibit the same 'weight'. Numerical experiments allowed to find out the following optimal ratios:

$$
h_{1} \cong \frac{1}{5} L_{1}, h_{2} \cong \frac{1}{50} L_{2}, \tau \cong\left(\frac{h_{1}}{L_{1}}\right)^{2} .
$$

We write down the locally unidimensional (longitudinal-transversal) scheme ('variable direction' implicit scheme) at the $(j+1)$ th time and for the $r$ th iteration in the following iterative form. Time discretization is given by $j=0,1, \ldots, N_{0}$.

The initial conditions at $t=0$ and at the beginning of the current time step ( $r=0$ iteration) are respectively expressed by

$$
\begin{gathered}
v_{(\alpha) i_{1} i_{2}}^{0}=\bar{\vartheta}\left(i_{1} h_{1}, i_{2} h_{2} ; 0\right), i_{1}=0,1, \ldots, N_{1}, i_{2}=0,1, \ldots, N_{2} \\
v_{(2) i_{1} i_{2}}^{j+1,0}=v_{(2) i_{1} i_{2}}^{j}, f_{1, i_{2}}^{j+1,0}=f_{1, i_{2}}^{j}, f_{2, i_{1}}^{j+1,0}=f_{2, i_{1}}^{j} .
\end{gathered}
$$

Space discretization in the coordinate direction $\alpha=1$ (longitudinal scheme) is given by

$$
i_{2}=1,2, \ldots, N_{2}-1, i_{1}=0,1, \ldots, N_{1} \text {. }
$$


The equation of thermal conduction in either the solid $(\varphi=1)$ and liquid $(\varphi=2)$ phase and the evolution equation of moving boundary between solid and liquid phases are respectively

$$
\begin{gathered}
\Lambda_{t}^{-} v_{(1) i, i_{2}}^{j+1, r}=\left(\frac{k}{\rho c}\right)_{\varphi}\left[\Lambda_{11} v_{(1) i i_{1}}^{j+1, r}+\Lambda_{22} v_{(2) i_{1} i_{2}}^{j+1, r-1}\right] \\
k_{s}\left[L_{(1)}^{-1} w_{(1) s_{1} i_{2}}^{+l, r}-\Lambda_{2}^{0} v_{(2) m_{1} i_{2}}^{++1, r-1} \Lambda_{2}^{0} f_{1, i_{2}}^{j+1, r-1}\right]-k_{1}\left[L_{(1) s, i_{2}}^{+1 v j+1, r}-\Lambda_{2}^{0} v_{(2) m_{1}}^{++1, r-1} \Lambda_{2}^{0} f_{1, i_{2}}^{j+1, r-1}\right]=c^{*} \Lambda_{t}^{-1} f_{1, i_{2}}^{j+1, r} \\
s_{1}=f_{1, i_{2}}^{j+1, r-1}, m_{1} h_{1} \leq s_{1} \leq\left(m_{1}+1\right) h_{1}, v_{(1) s_{1} i_{2}}^{j+l, r}=\vartheta^{*} .
\end{gathered}
$$

\subsection{Space discretization}

Space discretization in the coordinate direction $\alpha=2$ (transversal scheme) is given by

$$
i_{1}=1,2, \ldots, N_{1}-1, i_{2}=0,1, \ldots, N_{2} .
$$

The equation of thermal conduction in either the solid $(\varphi=1)$ or liquid $(\varphi=2)$ phase and the evolution equation of moving boundary between solid and liquid phases are respectively

$$
\begin{aligned}
& \Lambda_{t}^{-} v_{(2) i_{1} i_{2}}^{j+1, r}=\left(\frac{k}{\rho c}\right)_{\varphi}\left[\Lambda_{11} v_{(1) i_{i} i_{2}}^{j+1, r}+\Lambda_{22} v_{(2) i_{1} i_{2}}^{j+1, r}\right] \\
& k_{\mathrm{s}}\left[\Lambda_{1}^{0} v_{(1) i, m_{2}}^{j+1, r}-\Lambda_{1}^{0} f_{2, i_{1}}^{j+1, r-1}+L_{2}^{-} v_{(2) i, s_{2}}^{j+1, r}\right]-k_{l}\left[-\Lambda_{l}^{0} v_{(1) i, m_{2}}^{j+1, r}-\Lambda_{1}^{0} f_{2, i_{1}}^{j+1, r-1}+L_{2}^{+} v_{(2) i, s_{2}}^{j+1, r}\right]=c^{*} \Lambda_{t}^{-} f_{2, i_{1}}^{j+1, r} \\
& s_{2}=f_{2, i_{1}}^{j+1, r-1}, m_{2} h_{2} \leq s_{2} \leq\left(m_{2}+1\right) h_{2}, v_{(2) i i_{2}}^{j+1, r}=\vartheta^{*} \text {. }
\end{aligned}
$$

The solution at the $r$ th iteration and the convergence condition of the longitudinaltransversal scheme for $\alpha=1,2$ respectively read as follows:

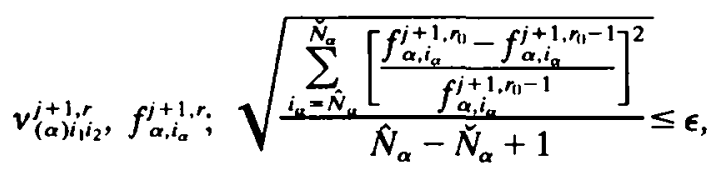

where $\epsilon$ is a suitable tolerance, and $\hat{N}_{\alpha}, \check{N}_{\alpha}$ are defined as follows:

$$
\check{N}_{2}=1, H_{(1)}^{i+1, r}-h_{2} \leq h_{2} \hat{N}_{2} \leq H_{(1)}^{j+1, r} ; 0 \leq f_{2, N_{1}}^{j+1, r} \leq h_{2}, H_{(2)}^{i+1, r}-h_{2} \leq f_{2, N_{1}}^{j+1, r} \leq H_{(2)}^{i+1, r} .
$$

When convergence is attained ( $r_{0}$ th iteration) we have

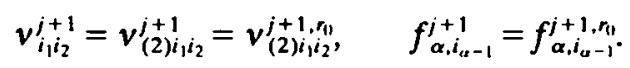

The current height of liquid head $H_{(\alpha)}^{j+1, r}$ has been evaluated by discretizing equation (3.3), which expresses mass conservation. The trapezoidal rule has been applied in order to calculate the current volume of solid phase.

\section{NUMERICAL DETERMINATION OF INGOT SHAPE FOR BOUNDARY CONDITIONS ENCOUNTERED IN ENGINEERING APPLICATIONS}

\subsection{Generalities}

A two-dimensional cast of rectangular shape has been considered which is initially filled with liquid aluminium (pure metal); thermophysical properties of the material have been shown in Table 1, while phase change data are given in Table 2.

Table 1. Thermophysical properties of aluminium [23]

\begin{tabular}{lccc}
\hline & $\begin{array}{c}\text { Mass density } \\
{\left[\mathbf{K g m}^{3}\right]}\end{array}$ & $\begin{array}{c}\text { Specific heat } \\
{\left[\mathrm{J}^{\circ} \mathbf{K}^{-1} \mathbf{K g}^{-1}\right]}\end{array}$ & $\begin{array}{c}\text { Thermal conductivity } \\
{\left[\mathbf{W}^{0} \mathbf{K}^{-1} \mathbf{m}^{-1}\right]}\end{array}$ \\
\hline $\begin{array}{l}\text { Liquid phase } \\
\text { Solid phase }\end{array}$ & 2385 & 1080 & 100 \\
\hline
\end{tabular}


Table 2. Change of phase [23]

\begin{tabular}{lc}
\hline Melting temperature $\left[{ }^{\circ} \mathrm{K}\right]$ & 933.5 \\
Formula weight $\left(W_{\mathrm{f}}\right)$ & $26.9815(\mathrm{see}[24])$ \\
Latent heat $\left[\mathrm{JKg}\right.$-atom $\left.{ }^{-1}\right]$ & $10.47 / W_{\cdot} \cdot 10^{+6}$ \\
Volume change during melting & 6.5 \\
$\Delta V_{\mathrm{m}}=\left[\left(V_{\text {tiquid }}-V_{\text {solid }}\right) / V_{\text {solid }}\right](\%)$ & \\
\hline
\end{tabular}

A uniform mesh has been adopted with $N_{1}=5$, and $N_{2}=60$. The initial temperature and the boundary condition at the FMB (below the liquid head) are respectively

$$
\begin{gathered}
v_{(\alpha) i_{1} i_{2}}^{0}=\bar{\vartheta}\left(i_{1} h_{1}, i_{2} h_{2} ; 0\right)=\vartheta^{*}, i_{1}=0,1, \ldots, N_{1}, i_{2}=0,1, \ldots, N_{2} \\
v_{(\alpha)}^{j+1}=\vartheta^{*}, \alpha=1,2 .
\end{gathered}
$$

Adiabatic boundary conditions have been assumed on the symmetry axis, liquid head and bottom respectively:

$$
\begin{aligned}
& -k_{\varphi} \Lambda_{1}^{-} v_{(1) N_{1} i_{2}}^{j+1, r}=q\left[\frac{1}{2} L_{1}, i_{2} h_{2} ;(j+1) \tau\right] \cdot n_{\Gamma\left(x_{1}=L, 2\right)}=0, n_{\Gamma\left(x_{1}=L, / 2\right)}=\{1,0\} \\
& -k_{l} \Lambda_{1}^{-} v_{(2) i_{1} \bar{H}}^{j+1, r}=q\left[i_{1} h_{1}, H_{(2)}^{j+1, r-1} ;(j+1) \tau\right] \cdot n_{\Gamma\left(x_{2}=\bar{H}\right)}=0, n_{\Gamma\left(x_{2}=\bar{H}\right)}=\{0,1\} \\
& -k_{\varphi} \Lambda_{1}^{+} v_{(2) i_{1}, j}^{j+1, r}=q\left[i_{1} h_{1}, 0 ;(j+1) \tau\right] \cdot n_{\Gamma\left(x_{2}=0\right)}=0, n_{\Gamma\left(x_{2}=0\right)}=\{0,-1\} .
\end{aligned}
$$

Here, $\bar{H}=H_{(1)}^{j+1, r}=$ height of liquid head at $t_{j+1}=(j+1) \tau$ at the $(r-1)$ th iteration.

Two cases have been investigated which differ for the boundary conditions assumed along the side: (1) given temperature uniform in the $\alpha=2$ direction, and (2) assigned heat flux. In both cases the FMB velocity has been restricted to have only one nonzero component, namely in the $\alpha=1$ direction. Such an assumption is exactly fulfilled in the first of the following study cases, on the basis of the boundary conditions and of the analytical solution given in Section 4.3, wheras it seems to be reasonable in the second example, and it has been validated by means of a suitable numerical test.

\subsection{Given temperature at mold sides}

In the first sample, the rectangle sizes are $L_{1}=0.2 \mathrm{~m}$ and $L_{2}=0.1 \mathrm{~m}\left(L_{2} / L_{1}=2\right)$.

The boundary condition at the mold sides is

$$
v_{(1) i_{2}}^{j+1}=\bar{\vartheta}\left[0, i_{2} h_{2} ;(j+1) \tau\right]=\bar{\vartheta}=50^{\circ} K\left(<\vartheta^{*}\right), \forall i_{2} .
$$

The boundary condition at the solidified frontier (above the liquid head) is

$$
\begin{gathered}
v_{(1) \bar{s}_{1} i_{2}}^{j_{2}}=\hat{\vartheta}_{1}\left[\bar{s}_{1}, i_{2} h_{2} ;(j+1) \tau\right]=\bar{\vartheta}-\left(\bar{\vartheta}-\vartheta^{*}\right) \frac{\operatorname{erf}\left(\frac{z}{2 a_{s}}\right)}{\operatorname{erf}\left(\frac{\alpha}{2 a_{s}}\right)}, \bar{s}_{1}+f_{1, i_{2}}^{\bar{j}_{2}}, j>\bar{j}_{i_{2}}, \\
\bar{j}_{i_{2}}: i_{2} h_{2}>H_{(1)}^{\bar{j}_{1}, r-1}, z=\alpha \sqrt{\frac{t^{j_{2}}}{t^{j+1}}}
\end{gathered}
$$

where $t^{\bar{i}_{2}}$ is the solidification time of the $x_{2}=i_{2} h_{2}$ mesh line, $\bar{s}_{1}$ is the $x_{1}$-ordinate of the 
solidified frontier at the $x_{2}=i_{2} h_{2}$ mesh line, and $H_{(1)}^{\bar{j}_{2}, r-1}$ is the height of liquid head at time $t^{\bar{j}_{2}}=\bar{j}_{i_{2}} \tau$ at the $(r-1)$ th iteration;

$$
\begin{gathered}
v^{\prime}{ }_{(2) i, \bar{s}_{2}}=\hat{\vartheta}_{2}\left[i_{1} h_{1}, \bar{s}_{2} ;(j+1) \tau\right]=\bar{\vartheta}-\left(\bar{\vartheta}-\vartheta * \frac{\operatorname{erf}\left(\frac{z}{2 a_{s}}\right)}{\operatorname{erf}\left(\frac{\alpha}{2 a_{s}}\right)}, \bar{s}_{2}+f_{2, i_{1}}^{\bar{j}_{1}}, j>\bar{j}_{i_{1}},\right. \\
\bar{j}_{i_{1}}: \bar{s}_{2}>H_{(2)}^{\bar{h}_{1}, r-1}, z=\alpha \sqrt{\frac{t^{\bar{j}_{1}}}{t^{j+1}}}
\end{gathered}
$$

where $t^{\bar{i}_{1}}$ is the solidification time of the $x_{1}=i_{1} h_{1}$ mesh line, $\bar{s}_{2}$ is the $x_{2}$-ordinate of the solidified frontier at the $x_{1}=i_{1} h_{1}$ mesh line, and $H_{(2)}^{j_{1}, r}$ is the height of the liquid head at time $t^{\bar{i}_{1}}=\bar{j}_{i} \tau$ at the $(r-1)$ th iteration.
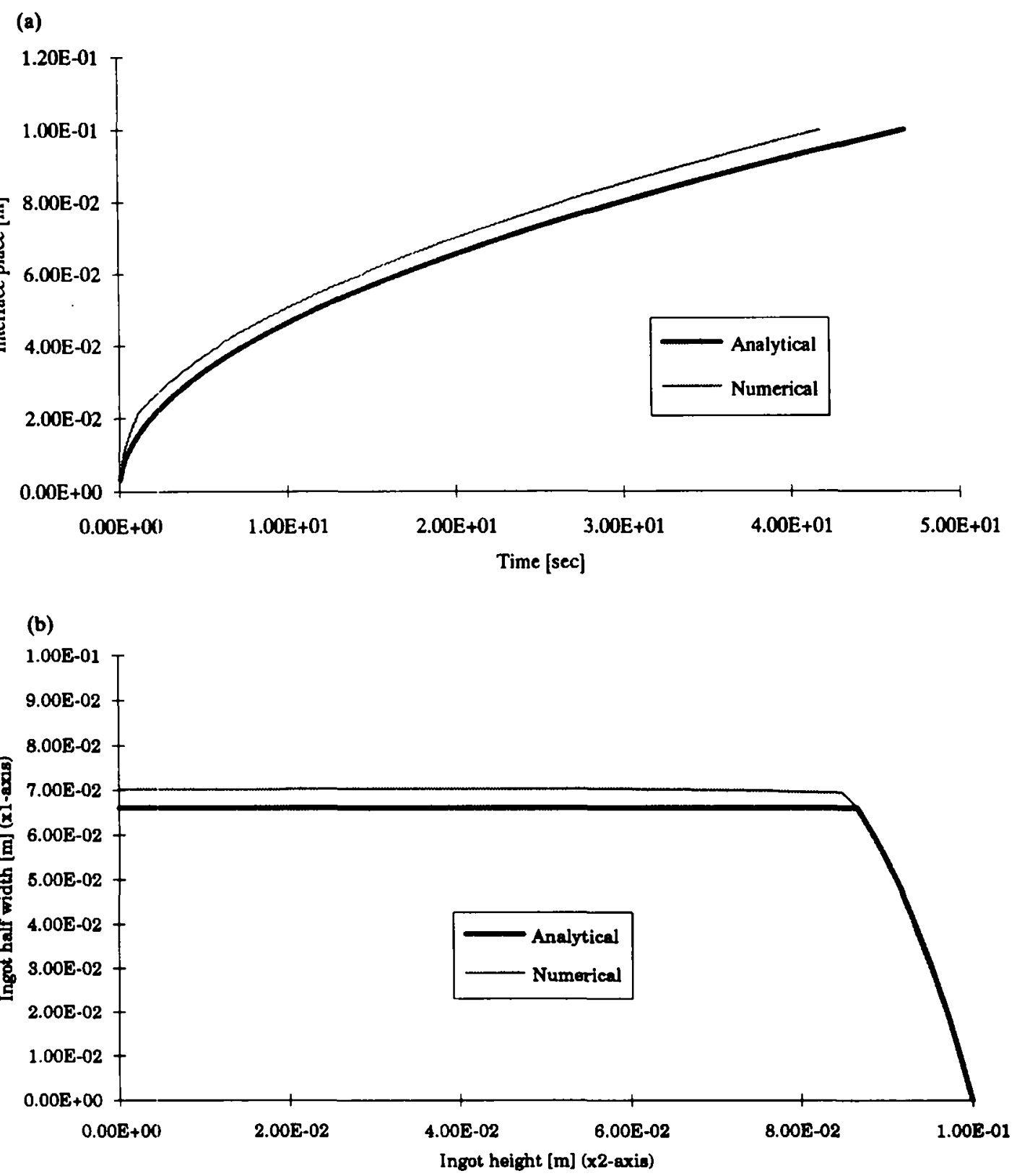

Fig. 2. (a) Solidification progress; (b) intermediate shapes for given temperature at mold sides at $t=20 \mathrm{sec}$. 
(a)

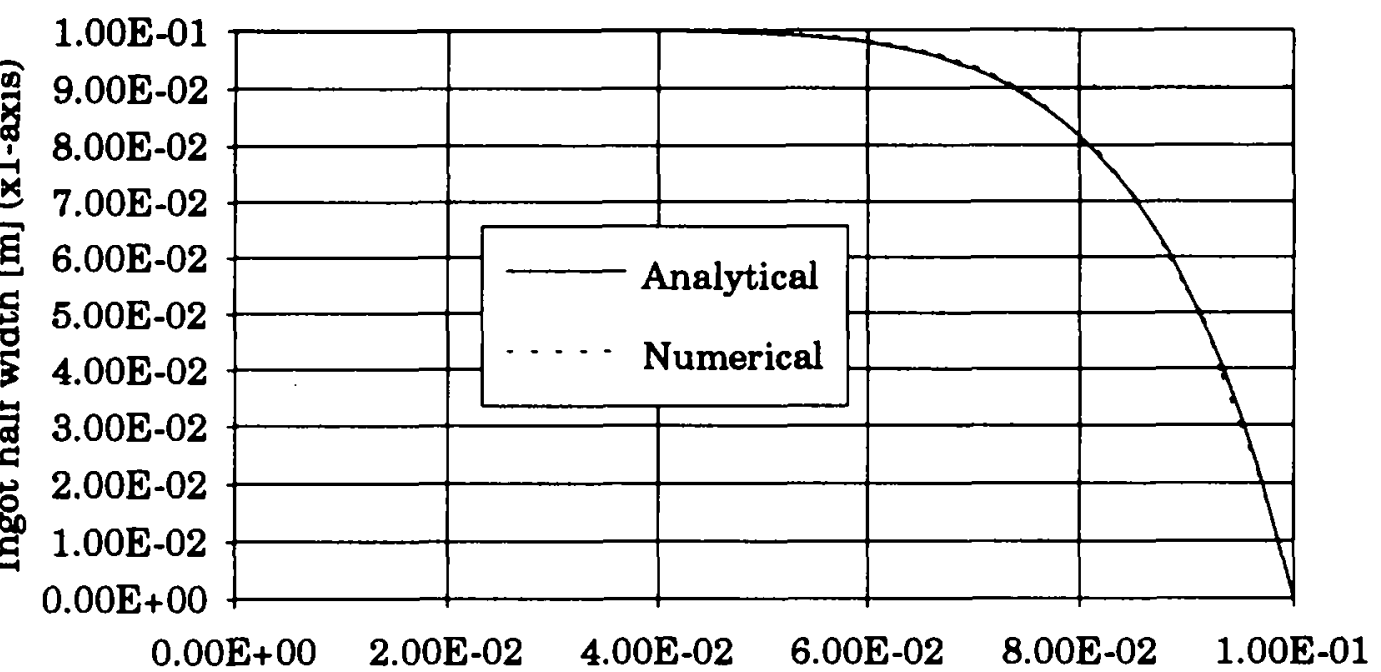

(b)

Ingot height $[\mathrm{m}](\mathbf{x} 2$-axis)

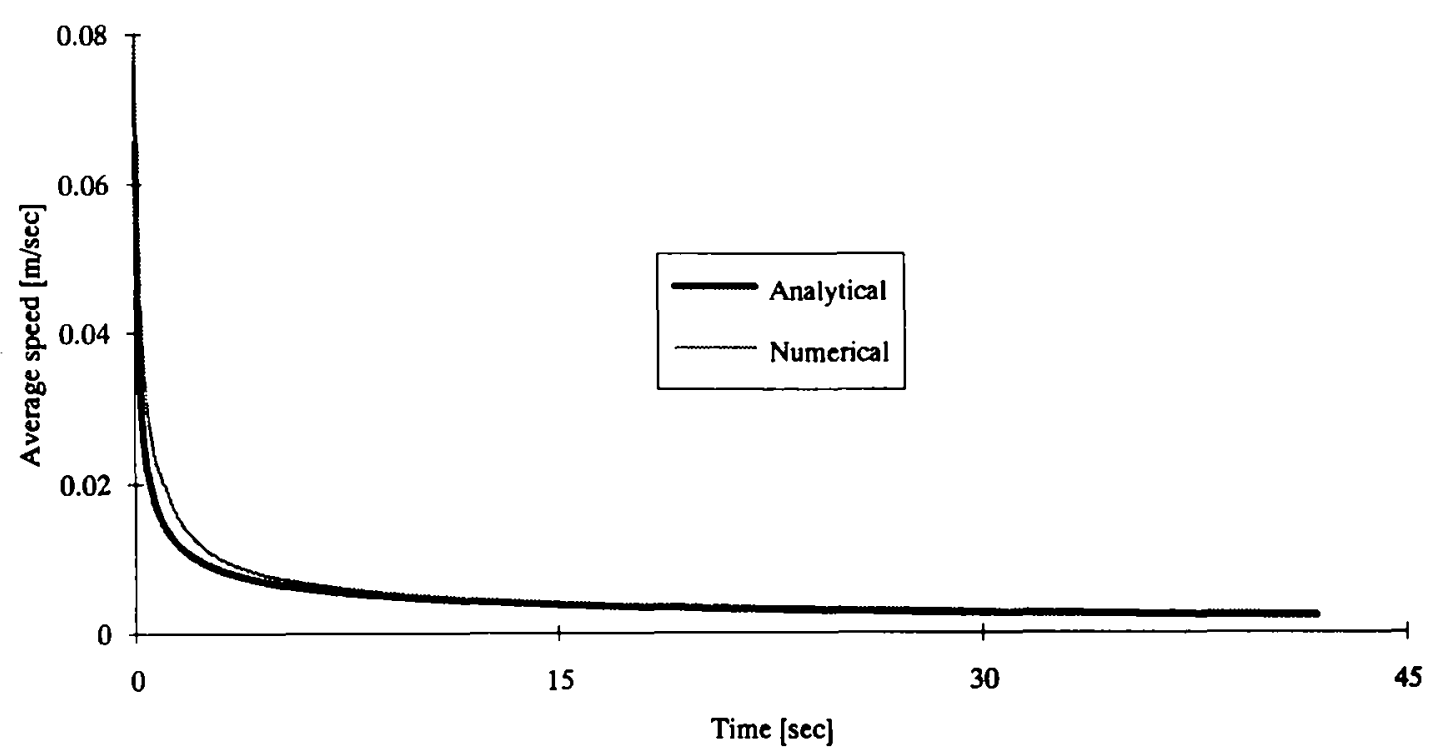

Fig. 3. (a) Final shapes for given temperature at mold sides; (b) time-histories of FMB average speed for given temperature at mold sides.

Introducing data of Table 1 and equation (8.1) in equation (4.7) and solving in respect to the solidification coefficient $\alpha$ gives the relevant value 0.0146623 . The total solidification time is equal to $41.8 \mathrm{sec}$, whereas its analytical forecast, obtained by equation (4.6) at $\xi=L_{1}$, is 46.5 sec. Comparison between analytical, equation (4.6), and numerical solidification progresses is made in Fig. 2(a). Figures 2(b) and 3(a) show the comparisons between (i) intermediate shapes (at $t=20 \mathrm{sec}$ ) and (ii) final shapes obtained in an analytical [equation (4.3)] (solid line) and numerical (thin line) way. Figure 3(b) shows the comparison between the time-histories of FMB average speed obtained in analytical, [equation (4.6) divided by $t$ ], (thick line) and numerical (thin line) way.

It is of value to note that the FMB does remain parallel to the $x_{2}$-axis throughout the solidification process (see Fig. 2(b)), as predicted by the analytical solution (see Section 4.3). Furthermore, temperature of the liquid phase is kept constant at melting value and temperature distribution in $x_{2}$-direction in solid phase does remain uniform. 
(a)

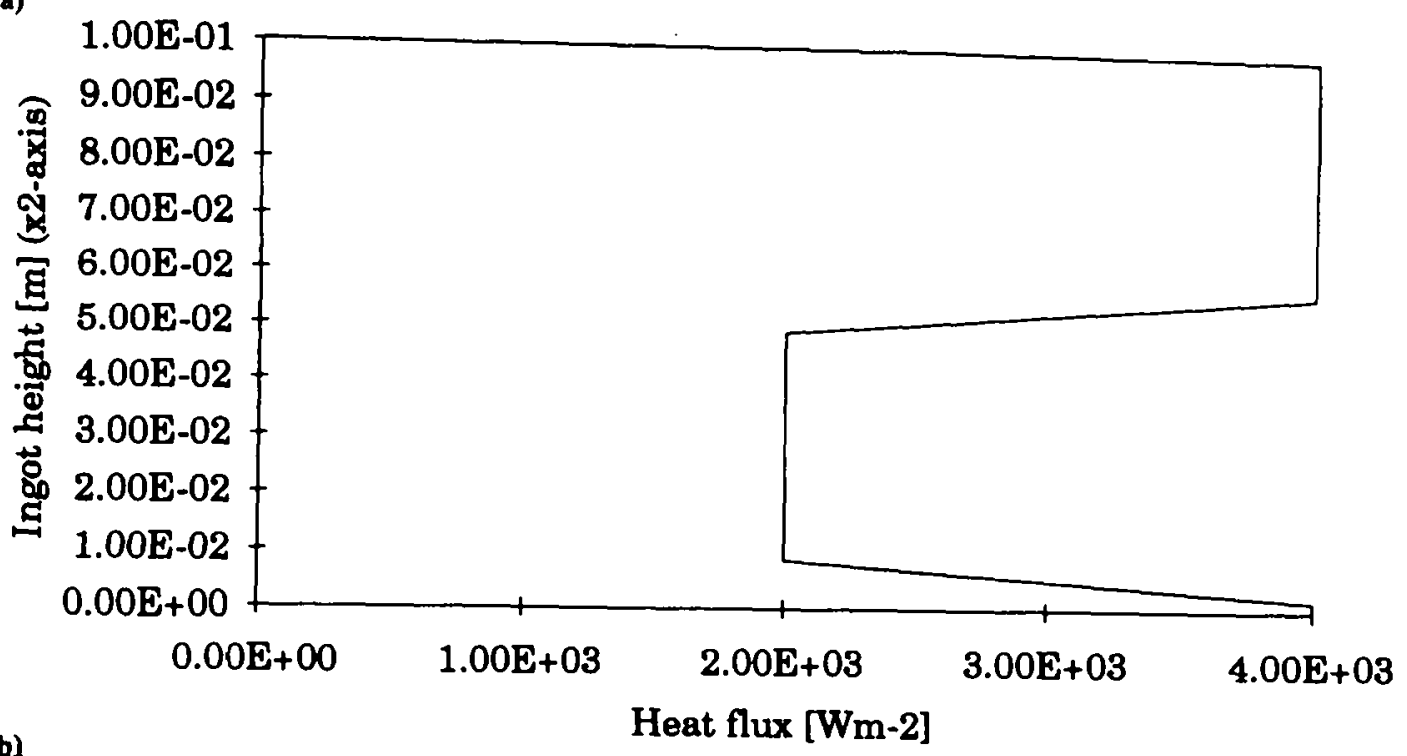

(b)

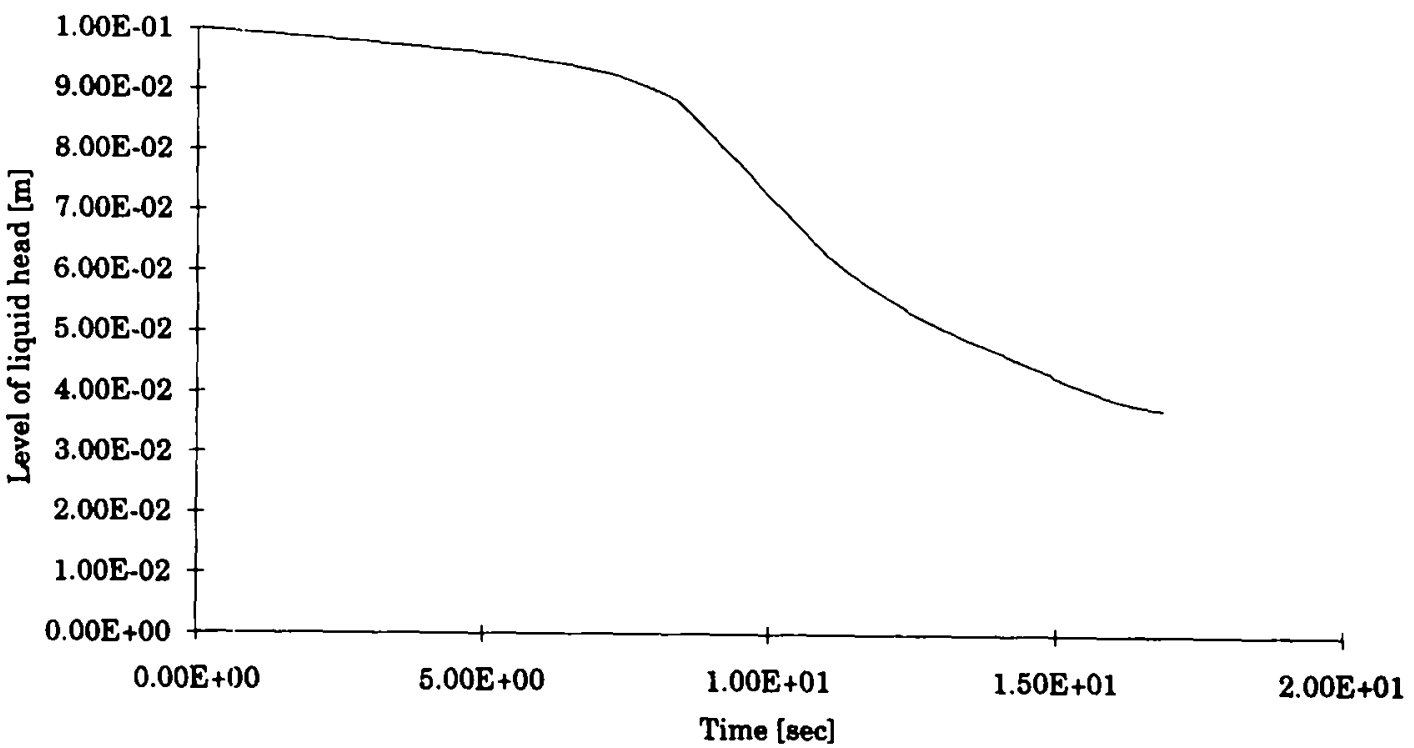

Fig. 4. (a) Heat flux given at mold sides; (b) time-history of liquid head level for given heat flux at mold sides.

\subsection{Given heat flux at mold sides}

In the second sample, the rectangle sizes are $L_{1}=0.0125 \mathrm{~m}$ and $L_{2}=0.1 \mathrm{~m}, L_{2} / 2 L_{1}=4$. The boundary conditions at the mold sides are (Fig. 4(a)):

$$
-k_{\varphi} \Lambda_{1}^{+} 1 v_{(1) 0 i_{2}}^{i+1, r}=q\left[0, i_{2} h_{2} ;(j+1) \tau\right] \cdot n_{\Gamma\left(x_{1}=0\right)}=\bar{q}_{i_{2}}, n_{\Gamma\left(x_{1}=0\right)}=\{-1,0\}, n_{\Gamma\left(x_{1}=2 L_{1}\right)}=\{1,0\} .
$$

The boundary condition at the solidified frontier above the liquid head is

$$
\begin{aligned}
& v_{(1) \bar{s}_{1} i_{2}}^{j}=\hat{\vartheta}_{1}\left(\bar{s}_{1}, i_{2} h_{2} ;(j+1) \tau\right)=\vartheta * \frac{\operatorname{erf}\left(\frac{z}{2 a_{s}}\right)}{\operatorname{erf}\left(\frac{\alpha}{2 a_{s}}\right)}, \bar{s}_{1}=f_{i_{, l_{2}}}^{\dot{i}_{2}}, j>\bar{j}_{i_{2}}, \\
& \bar{j}_{i_{2}}: i_{2} h_{2}>H_{(1)}^{\bar{j}_{i_{1}, r-1}}, \quad z=\frac{\overline{s_{1}}}{\sqrt{t^{j}}}, \alpha=\frac{\bar{s}_{1}}{\sqrt{\bar{t}_{j_{2}}}} ;
\end{aligned}
$$


(a)

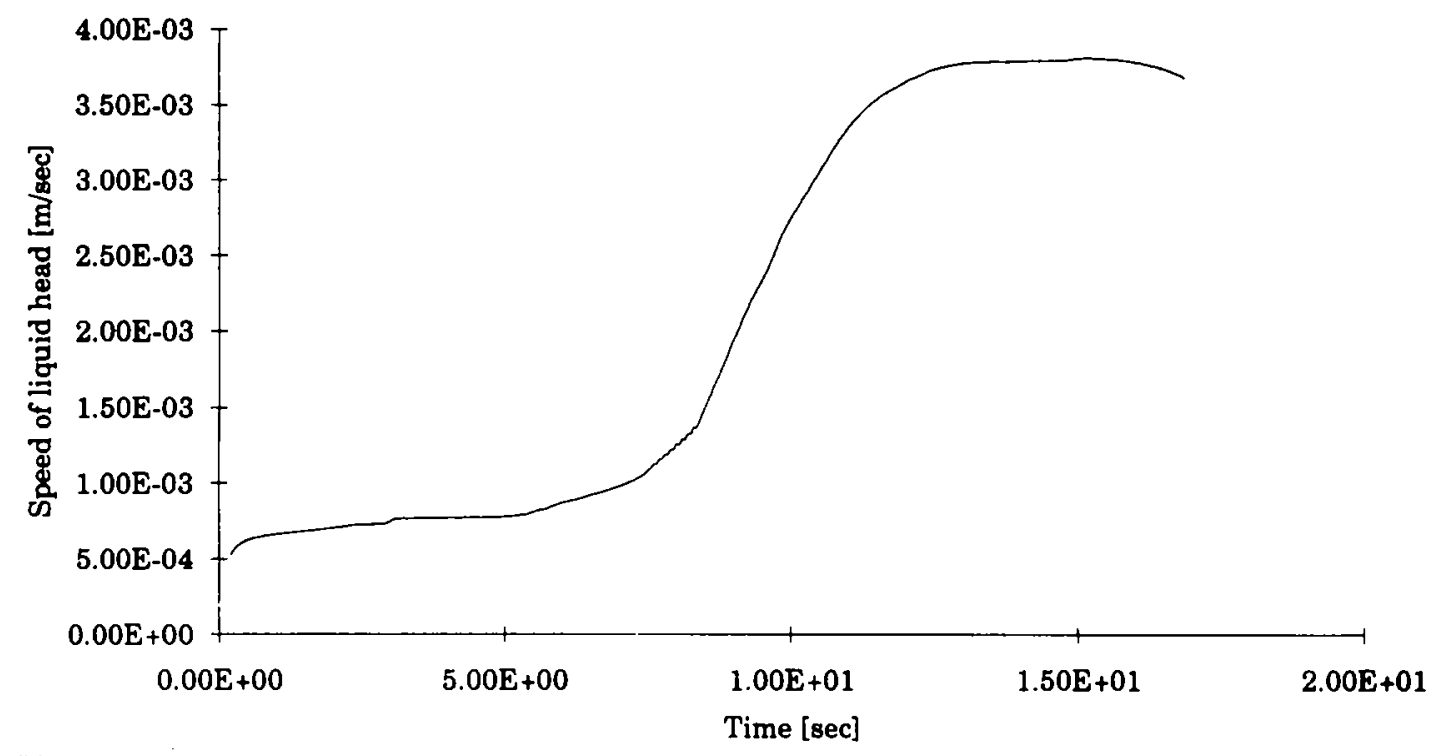

(b)

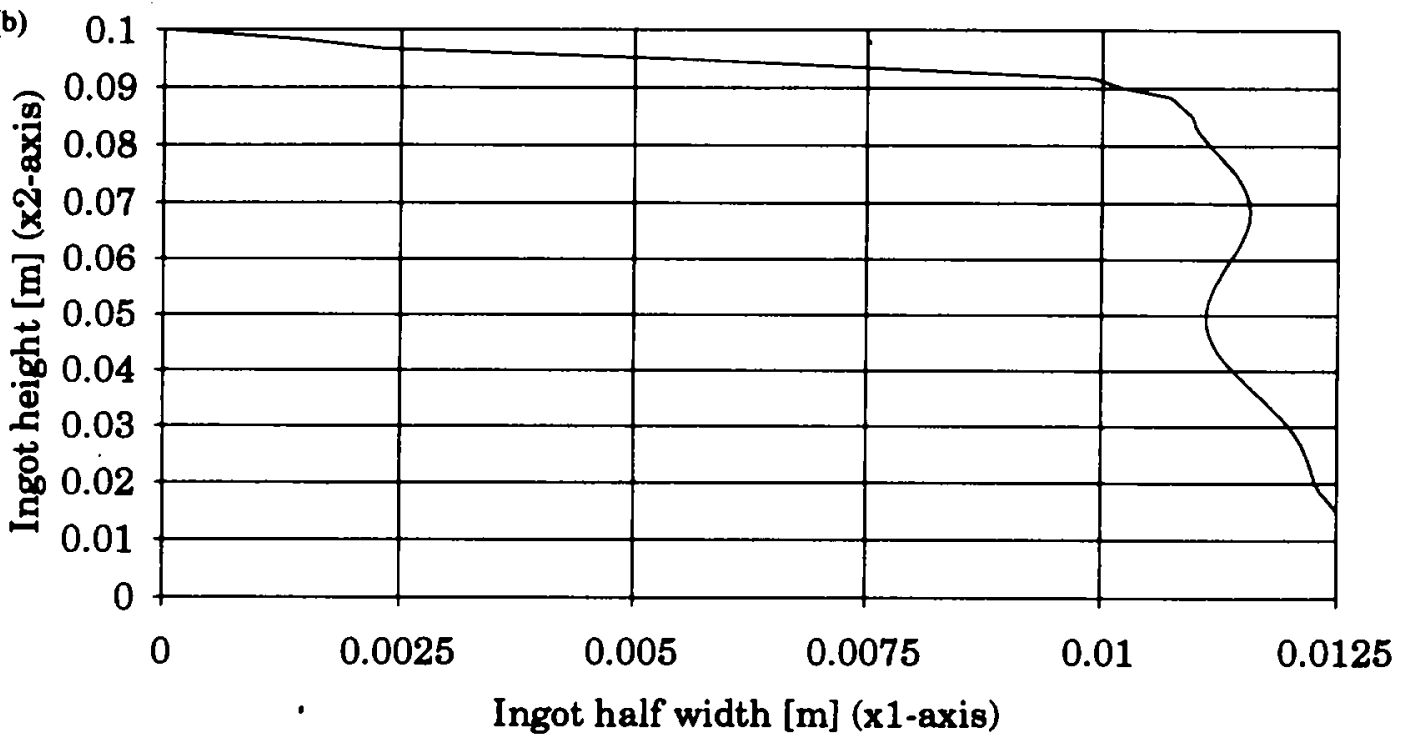

Fig. 5. (a) Time-history of liquid head average speed for given heat flux at mold sides; (b) final shape for given heat flux at mold sides.

where $t^{\bar{i}_{2}}$ is the solidification time of the $x_{2}=i_{2} h_{2}$ mesh line, $\bar{s}_{1}$ is the $x_{1}$-ordinate of the solidified frontier at the $x_{2}=i_{2} h_{2}$ mesh line, and $H_{(1)}^{j_{1}, r-1}$ is the height of liquid head at time $t^{\bar{i}_{2}}=\bar{j}_{i_{2}} \tau$ at the $(r-1)$ th iteration;

$$
\begin{aligned}
& v_{(2) i_{1} \bar{s}_{2}}^{j}=\hat{\vartheta}_{2}\left(i_{1} h_{1}, \bar{s}_{2} ;(j+1) \tau\right)=\vartheta * \frac{\operatorname{erf}\left(\frac{z}{2 a_{s}}\right)}{\operatorname{erf}\left(\frac{\alpha}{2 a_{s}}\right)}, \bar{s}_{2}+f_{2, i_{1}}^{\bar{i}_{1}}, j>\bar{j}_{i_{1}}, \\
& \bar{j}_{i_{1}}: \bar{s}_{2}>H_{r(2)}^{\bar{i}_{1},}, \quad z=\frac{\bar{s}_{2}}{\sqrt{t^{\prime}}}, \alpha=\frac{\bar{s}_{2}}{\sqrt{t^{j_{1}}}}
\end{aligned}
$$

where $t^{\bar{j}_{11}}$ is solidification time of the $x_{1}=i_{1} h_{1}$ mesh line, $\bar{s}_{2}$ is the $x_{2}$-ordinate of the solidified frontier at the $x_{1}=i_{1} h_{1}$ mesh line, and $H_{(2)}^{\bar{i}_{1}, r}$ is the height of liquid head at time $t^{\bar{j}_{1}}=\bar{j}_{i_{1}} \tau$ at the $(r-1)$ th iteration. 
The total solidification time is $16.9 \mathrm{sec}$. Figures 4(b) and 5(a) illustrate time-histories of level and average speed of liquid head respectively; Fig. 5(b) shows the final shape obtained in the numerical approach.

A simple test has been successfully performed in order to validate the assumption of null 2-component of FMB velocity: the maximum value of the ratio between heat flux components through the FMB has been numerically evaluated at each time according to

$$
q_{\alpha}\left(\xi_{\alpha-1} ; t\right)=-k_{s} \frac{\partial \vartheta}{\partial x_{\alpha}}
$$

where the temperature space rate has been approximated by $L_{1}^{-}[14]$ and $\Lambda_{2}^{0}$ [20], operators in the 1 - and 2-directions respectively. This maximum ratio remained less than a few percent.

\section{REFERENCES}

[1] B. CHALMERS, Principles of Solidification. R. E. Krieger Publishing Company, Malabar, Florida (1982).

[2] J. SZEKELY, Some mathematical, physical and engineering aspects of melting and solidifications problems. In Free Moving Boundary Problems: Theory and Applications (Edited by A. FASANO and M. PRIMICERIO), Vol. I. Pitman (1983).

[3] F. DELL'ISOLA and A. ROMANO, Int. J. Engng Sci. 25, 1459 (1987).

[4] F. DELL'ISOLA and A. ROMANO, Int. J. Engng Sci. 25, 1469 (1987).

[5] C. TRUESDEL.L, Rational Continuum Mechanics (2nd Edn). Academic Press (1992).

[6] W. NOLL, The Foundation of Mechanics and Thermodynamics, Selected Papers. Springer-Verlag (1974).

[7] A. FRIEDMAN, Trans. Amer. Math. Soc. 133 (1968).

[8] J. CRANK, Free and Moving Boundary Problems. Clarendon Press, Oxford (1984).

[9] B. MOCHNAC:KI and M. BALCER, Solidification problem. Numerical model on the basis of generalized FDM. In Proc. of the 8th Int. Conf. on Numerical Methods in Thermal Problems (Edited by R. W. LEWIS), Part 1, pp. 329-339, Swansea, UK, July 12-16 (1993).

[10] D. V. TRAN, R. W. LEWIS and D. T. GETHIN, Multi-adaptative finite element remeshing with specific reference to metal casting. In Proc. of the 8th Int. Conf. on Numerical Methods in Thermal Problems (Edited by R. W. LEWIS), Part 2, pp. 955-970 Swansea, UK, July 12-16 (1993).

[11] A. R. MITCHELL and R. WAIT, The Finite Element Method in Partial Differential Equations. John Wiley and Sons, Chichester (1978).

[12] R. D. RICHTMYER and K. W. MORTON, Difference Methods for Initial-Value Problems. John Wiley and Sons, Chichester (1967).

[13] T. M. SHIH, Numerical Heat Transfer. Series in Computational Methods in Mechanics and Thermal Sciences. Editions Hemisphere, Washington (1984).

[14] J. CRANK, Proc. of the Conf. 'Moving boundary problems in heat flow and diffusion, pp. 192-207, Oxford, 25-27 March (1974).

[15] A. LAZARIDIS, Int. J. Heat Mass Transfer 13, 1459 (1970).

[16] J. HLOUSEK and F. KAVICKA, A coherence between a castings shape and its total solidification time. In Proc. of the 2nd Int. Conf. on Computational Modelling of Free and Moving Boundary Problems (Edited by L. C. WROBEL and C. A. BREBBIA), pp. 347-356, Milan, June (1993).

[17] B. A. BOLEY and J. H. WEINER, Theory of Thermal Stresses. John Wiley and Sons, New York (1960).

[18] A. FASANO and M. PRIMICERIO (Eds), Free Moving Boundary Problems: Theory and Applications, Vol. I. Pitman (1983).

[19] L. C. WROBEL and C. A. BREBBIA (Eds), Computational Modelling of Free and Moving Boundaries II. Computational Mechanics Publications, Southampton, MA (1993).

[20] A. N. TICHONOV and A. A. SAMARSKIJ, Equazioni della Fisica-matematica. Edizioni Mir, Mosca (1981).

[21] J. J. DONGARRA, J. R. BUNCH, C. B. MOLER and G. W. STEWART, LINPACK Users' Guide. SIAM, PA (1979).

[22] A. K. CLINE, C. B. MOLER, G. W. STEWART, and J. H. WILKINSON. SIAM Journal of Numerical Analysis 16, 368 (1979).

[23] E. A. BRANDES, Smithells Metals Reference Book. Butterworth and Co. Publishers Ltd, London (1983).

[24] R. C. WEAST, CRC Handbook of Chemistry and Physics. CRC Press Inc., Boca Raton, FL (1985). 